\title{
Nachruf auf Klaus Zerinschek (1946-2019)
}

Haiku für den Lehrer

Er breitet sein Wissen aus Und wartet. Das ist seine Kraft.

So lernt der Schüler ${ }^{1}$

Klaus Zerinschek wurde am 21.12.1946 in Innsbruck geboren. Nach der Reifeprüfung am Akademischen Gymnasium in Innsbruck 1966 und anschließendem Militärdienst nahm er das Studium (Germanistik, Geschichte, Kunstgeschichte) an der Geisteswissenschaftlichen Fakultät der Leopold-Franzens-Universität Innsbruck auf und schloss es mit der Lehramtsprüfung (Deutsch und Geschichte) und der Sponsion zum Magister der Philosophie ab. Er war einer der ersten Studenten des im Jahr 1970 neugegründeten Fachs der Vergleichenden Literaturwissenschaft an der Universität Innsbruck, das zu jener Zeit noch an die Germanistik angebunden war. Vergleichen, ergründen, über den Rand der traditionellen Disziplinen hinausblicken, waren von jeher zentrale Anliegen von Klaus Zerinschek. So setzte er sein Studium in den Fächern Anglistik, Amerikanistik, Musikwissenschaft und Vergleichende Literaturwissenschaft fort und promovierte 1981 zum Doktor der Philosophie. Der Titel seiner Dissertation war Jakob Michael Reinhold Lenz' Werke auf dem modernen Musiktheater. Ein Beitrag zur Strukturbestimmung des Opernlibrettos. Er habilitierte sich 1990 mit dem Thema Paradigmen zur Untersuchung des Opernlibrettos nach literarischen Vorlagen. Ein Beitrag zur Theoriebildung der Vergleichenden Literaturwissenschaft und erhielt die Lehrbefugnis als Universitätsdozent für Vergleichende Literaturwissenschaft. Die Liebe zur Oper und zum Theater hat sein Leben besonders geprägt - sagte er doch am Vorabend seines Todes „Jetzt hat der Engel der Musik das Ruder übernommen."

Seine berufliche Tätigkeit an der Universität Innsbruck begann Klaus Zerinschek 1972 als Studienassistent an der Vergleichenden Literaturwissenschaft; er wirkte dort zusammen mit Prof. Zoran Konstantinović und Fridrun Rinner maßgeblich am Aufbau und an der Einrichtung des ersten unabhängigen Instituts für Vergleichende Literaturwissenschaft in Österreich mit. 1977 wurde er Universitätsassistent und 1990 schließlich Assistenzprofessor. Er war von 1999 bis 2000 Institutsvorstand und nach der Fusion der Institute für Vergleichende Literaturwissenschaft, Sprachwissenschaft und Klassische Philologie zum „Institut für Sprachen und Literaturen“ von 2000 bis 2009 stellvertretender Institutsvorstand. 
Während dieser Zeit beteiligte er sich auch an der Organisation von zahlreichen wissenschaftlichen Symposien, bei denen immer die aktuellsten Themen der Komparatistik (Literaturtheorie, der mitteleuropäische Kulturraum und Interdisziplinarität) im Mittelpunkt standen. Ganz besonders bedeutsam war die Ausrichtung des IX. Weltkongresses der AILC (Association Internationale de Littérature Comparée) im Jahre 1979, bei dem erstmals die Forschungsrichtung „Literatur und andere Künste“ als eigenes Forschungsgebiet der Komparatistik eingeführt wurde. ${ }^{2}$

Von diesem Zeitpunkt an machte Klaus Zerinschek Intermedialität und das komplexe Relationsgeflecht zwischen Literatur und anderen Kunstformen, wie Malerei, Architektur, Tanz, Musik, Fotografie, Film, Performance-Art und digitaler Kunst, zu zentralen Lehrund Forschungsschwerpunkten am Innsbrucker Institut für Vergleichende Literaturwissenschaft. Durch zahlreiche Forschungsaufenthalte erweiterte er kontinuierlich seinen wissenschaftlichen Horizont (Indiana University, Bloomington; University of California, Berkely; New York University, N.Y.; University of Michigan, Ann Arbor; Columbia University, N.Y.; University of Connecticut, CT; Rice University Houston; Studienaufenthalt in London). Opernbesuche und Kunstevents durften natürlich nie fehlen und eine Karte für die Metropolitan Opera war ihm wichtiger als das Essen. Auch für den sukzessiven Ausbau seiner faszinierend reichhaltigen Bibliothek, die insbesondere zu den Beziehungen von Literatur und anderen Formen des künstlerischen Ausdrucks ganz seltene Werke umfasste, waren seine zahlreichen Auslandsaufenthalte und Studienreisen eine wichtige Quelle.

Seine Begeisterung für Oper, Musik und Theater hat er - Theorie und Praxis verbindend an viele Studierende weitergegeben, er besuchte gemeinsam mit ihnen Aufführungen und Museen und organisierte mehrere Exkursionen. Eine dreiwöchige, gemeinsam geplante Reise führte Studierende und Institutsmitglieder in die ehemalige DDR, nach Prag, Budapest, Zagreb und Triest, andere Exkursionsziele waren u.a. Galizien und das Baltikum.

Generationen von Studierenden besuchten seine innovativen und breit gefächerten Lehrveranstaltungen. Anita Moser, eine seiner ehemaligen Student*innen, schrieb anlässlich seines Ruhestandes:

[...] man wundert sich nicht nur, wo er das immense Wissen, das noch dazu stets am letzten Stand gehalten wird, herhat [sic], sondern auch, wo er wohl diesen außergewöhnlichen Fotoband schon wieder auftreiben konnte oder jenes noch druckfrische Buch zur neuesten Kunstdebatte, wie er diese leicht krachenden Originalstimmen oder jenen dermaßen raren Filmmitschnitt ergattert hat. ${ }^{3}$

Von diesem Wissensdrang und seiner Vermittlung zeugen auch seine Vorlesungen und Seminare, die sich mit Themen auseinandersetzten wie: „Oper und Politik“; „Vom Neuwerden des Alten: Operninszenierungen und Intermedialität“; „Literatur und Malerei“; „Ästhetik und Gewalt“; „'Lustort Ohr': Stimmen/Ton/Sprache/Musik im 'neuen Hörspiel'“; „'Stimmen hören': Interart in den Medien“; „Heavy Metal' und Barockoper - Literatur, Oper, Popart“; „Kulturlandschaft lesen: Das Baltikum als Kulturraum“ (mit Exkursion) oder „Die 
'Stadt' als Chiffre in Literatur, Photographie, Film und Oper“. Klaus Zerinschek hat über 130 Diplomarbeiten und 16 Dissertationen zu den verschiedensten Themen aus seinen Lehrveranstaltungen betreut.

„Doch nicht nur inhaltlich begeistert Klaus Zerinscheks Unterricht“ - so schreibt Anita Moser-,

sondern vor allem auch durch die Art und Weise, wie die Inhalte dargeboten werden und die Auseinandersetzungen darüber stattfinden können: offen, lustvoll, kontroversiell, skeptisch, forschend, herausfordernd und das alles in einer Atmosphäre, die durchgehend von seinem wohlwollenden Interesse den Menschen und deren Fragen und Meinungen gegenüber - und oft auch vom umwerfenden Duft frisch gebrühten türkischen Kaffees - durchdrungen ist. ${ }^{4}$

In Innsbruck und an vielen anderen Orten im In- und Ausland leben und arbeiten Absolvent*innen der Vergleichenden Literaturwissenschaft, die Klaus Zerinschek mit seinem enzyklopädischen komparatistischen Wissen, seiner Menschlichkeit, seiner Achtsamkeit, seiner kritischen Perspektive und seinem gesellschaftspolitischen Engagement geprägt hat und die das bei und von ihm Erfahrene und Gelernte auf ihre Weise weitertragen.

Astrid Gostner, ehemalige Studentin, charakterisiert „die hohe Kunst der Lehre des Klaus Zerinschek“:

[Den] grundlegende[n] und selbstverständliche[n] Respekt vor jedem Gedanken und jeder Meinung, das sensible Hinführen des ursprünglich menschlichen Forschertriebes zum wissenschaftlichen Arbeiten und die Fähigkeit, sich die kindliche Neugier und Offenheit zu bewahren, fern jeder Besserwisserei, die sich so gerne nach jahrelangem Forschen und Lehren einstellt. Diese Haltung erst bereitet den Boden für die Freude zum [sic] Lernen, Erkennen und Suchen, verwandelt Lehrveranstaltungsräume in Labors und gibt die Sicherheit, dass es sich lohnt, den eigenen Theorien auf den Grund zu gehen, sie am Empirischen zu erproben und sie mit anderen Theorien in Dialog zu führen. ${ }^{5}$

Seine Menschlichkeit und Begeisterungsfähigkeit kommen auch in den Worten seiner Studierenden anlässlich seines Todes zum Ausdruck. In den sozialen Netzwerken wird er als „der Inbegriff eines guten Literaturprofessors, der seine Liebe zur Literatur mit Begeisterung vermitteln konnte“ bezeichnet. Jemand schreibt: „Er war ein begeisterter und begeisternder Professor, mit Wissen und Zuwendung zum Fach und zu den Büchern“; oder „neben all den fachlichen und inhaltlichen Dingen, die wir von ihm gelernt haben, bleibt mir immer die Begeisterung hängen, die Klaus allem und jedem/jeder entgegengebracht hat, seine Menschlichkeit“, „er forderte Begeisterungsfähigkeit, Genauigkeit - und den Mut, tatsächlich zu denken“. „Manchen studentischen Deutungsexzess“ - schreibt Joachim Leitner, Journalist und ehemaliger Student von Klaus Zerinschek, im Nachruf in der Tiroler 
Tageszeitung vom 20. Februar 2019 - „ertrug er mit bewundernswerter Nachsicht (...). Noch scheint es unvorstellbar, ihn bei grandiosen oder grandios gescheiterten Konzerten, bei Tanz-, Theater- und - vor allem - Opernpremieren nicht mehr irgendwo im Saal zu wissen." 6

Trotz des Lebens- und Arbeitsmittelpunkts in Innsbruck blieb Klaus Zerinschek seiner Heimat, der in der Nähe von Innsbruck gelegenen Gemeinde Mieders im Stubaital, bis zu seinem Tod eng verbunden. Im Sommer wie im Winter zog es ihn in die Berge. Ihm, dem Wander- und Musikfreund, mögen die Worte aus Gustav Mahlers „Lied von der Erde“ gelten:

Wohin ich geh?? Ich geh', ich wandre in die Berge. [...]

Ich wandle nach der Heimat, meiner Stätte! [...]

Still ist mein Herz und harret seiner Stunde!

Die liebe Erde allüberall blüht auf im Lenz und grünt aufs neu!

Allüberall und ewig, ewig blauen licht die Fernen,

ewig, ewig [...]

Fridrun RINNER (Aix-en-Provence) / Magnus ROTH (Igls)

\section{Endnoten}

1 星歌手 hoshí joséi-káshu: „Haiku für den Lehrer“, in: KOMPlex. Literaturzeitschrift der Vergleichenden Literaturwissenschaft (Sonderausgabe 2009), 27. Dieses Haiku widmete Ulrike SöllnerFürst, eine seiner ehemaligen Student*innen, Klaus Zerinschek, als er 2009 in den Ruhestand ging.

2 Cf. den Kongressband Steven P. Scher / Ulrich Weisstein, Literature and the Other Arts, Innsbruck, Institut für Sprachwissenschaft der Universität Innsbruck, 1981.

3 Anita Moser: ," ${ }^{1}$ : Klaus Zerinschek ${ }^{2}$ geht $^{3}$ in Pension ${ }^{4 “}$ [sic], in: KOMPlex. Literaturzeitschrift der Vergleichenden Literaturwissenschaft (Sonderausgabe 2009), 7.

4 Anita Moser: ", Klaus Zerinschek ${ }^{2}$ geht $^{3}$ in Pension ${ }^{4 \text { “ }}$ [sic], in: KOMPlex. Literaturzeitschrift der Vergleichenden Literaturwissenschaft (Sonderausgabe 2009), 7.

5 Astrid Gostner: „Die Lehre“, in: KOMPlex. Literaturzeitschrift der Vergleichenden Literaturwissenschaft (Sonderausgabe 2009), 26.

6 jole [Joachim Leitner]: „Bilder, Beats und Pixel. Der Innsbrucker Komparatist Klaus Zerinschek starb im 73. Lebensjahr“, in: Tiroler Tageszeitung (20. Februar 2019), 17. 